\title{
Belphégor
}

\section{Yousif, Keri. Balzac, Grandville, and the Rise of the Book Illustration}

\section{Chris Reyns-Chikuma}

\section{(2) OpenEdition}

1 Journals

\section{Electronic version}

URL: http://journals.openedition.org/belphegor/725

DOI: $10.4000 /$ belphegor.725

ISSN: 1499-7185

\section{Publisher}

LPCM

\section{Electronic reference}

Chris Reyns-Chikuma, "Yousif, Keri. Balzac, Grandville, and the Rise of the Book Illustration », Belphégor [Online], 14 | 2016, Online since 10 October 2016, connection on 24 September 2020. URL : http://journals.openedition.org/belphegor/725 ; DOI : https://doi.org/10.4000/belphegor.725

This text was automatically generated on 24 September 2020

\section{(c) (i) (9)}

Belphégor est mis à disposition selon les termes de la Licence Creative Commons Attribution - Pas d'Utilisation Commerciale - Pas de Modification 4.0 International. 


\title{
Yousif, Keri. Balzac, Grandville, and the Rise of the Book Illustration
}

\author{
Chris Reyns-Chikuma
}

\section{REFERENCES}

Keri, Yousif, Balzac, Grandville, and the Rise of the Book Illustration, 2012

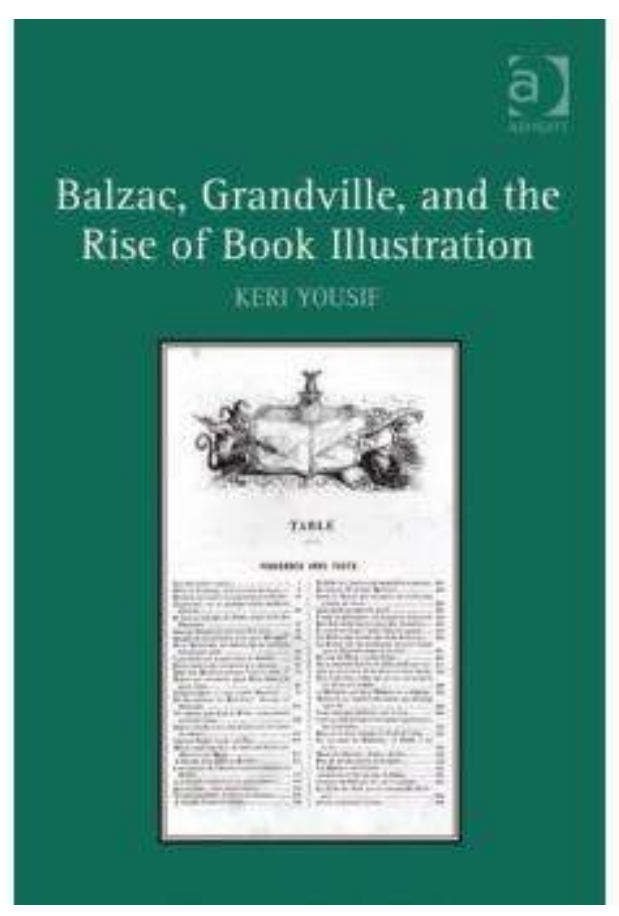

On pourrait croire que la mise en question de la dominance du texte par l'image date d'il y a quelques années avec l'internet ou tout au plus quelques décennies avec la télévision. Cependant, sans vouloir remonter à Platon, on pourrait citer les iconoclastes, la Réforme, le Romantisme avec le Laocoon qui attribuait des rôles très 
précis à la poésie et à la peinture pour éviter que ces deux arts ne se disputent leur territoire. Mais c'est sans aucun doute au début du $19^{\mathrm{e}}$ siècle que s'intensifie la concurrence entre ces deux médias.

Dans sa remarquable étude, Keri nous montre que la question n'est pas seulement idéologique mais aussi financière, symbolique, culturelle, hégémonique. À travers l'exemple de la compétition entre Balzac et Grandville, il nous montre comment cette rivalité professionnelle devenue personnelle est transposée dans leurs œuvres. Après avoir collaboré, Grandville parodiera les écrivains en général et Balzac en particulier dans Les Scènes de la vie privée et publique des animaux, et Balzac se moquera du caricaturiste et de l'illustrateur Grandville dans la Comédie humaine à travers le personnage récurrent de Bixiou. À partir d'exemples précis pris dans les œuvres de ces deux auteurs (des citations de La Comédie humaine et des dessins de Grandville), Keri étudie avec finesse cette compétition. L'analyse des dessins de Grandville est particulièrement subtile et instructive.

3 Dans son introduction, il montre d'abord comment, la censure ayant été abolie après la Révolution de juillet 1830, les cinq années de liberté ont suffi pour que la caricature fleurisse et gagne le crédit nécessaire pour devenir un art. Grâce aux avances technologiques (lithographie, presses mécanisées) et aux campagnes d'alphabétisation commencées avant la révolution, le prix du journal descend et le nombre des lecteurs (statistiquement majoritairement masculins) augmente, ce qui mènera à la création du fameux quotidien La Presse de Girardin en 1836. Le succès de ce journal apolitique fut en partie dû au roman-feuilleton avec ses techniques spécifiques (action, accroche, dialogues, ...) et au nouveau pouvoir donné à ses lecteurs de masse. Moins connu est le sort de la caricature. À la suite de la tentative d'assassinat de Louis-Philippe en 1835 une série de lois limitant la liberté de la presse est imposée. Plus que les mots, les images étaient craintes. Seul Le Charivari survivra à ces nouvelles lois et un marché noir d'images se développera. Mais le vrai changement est surtout dans le fait que la caricature politique se transformera alors en caricature sociale, beaucoup moins chargée, et en illustration des classiques et des bestsellers. On fait dès lors la satire des bourgeois qui votent plutôt que celle des hommes au pouvoir. Ces images deviennent tellement populaires que les éditeurs les reprennent en album. Elles gagnent en légitimité en devenant livre, soit de fiction, soit scientifique, soit pédagogique. Certains écrivains rejetèrent l'illustration au nom de l'art pur. D'autres, comme Balzac, ne pouvaient simplement pas se le permettre. Ils se voyaient dans l'obligation de collaborer et donc en même temps entraient en compétition avec l'illustrateur. En passant de caricaturiste à illustrateur, l'artiste s'assure un revenu régulier et une place parmi les grands de la littérature. L'étape suivante sera marquée par son accession à l'autonomie complète, faisant pour un temps basculer le pouvoir vers l'image et son artiste.

4 Dans son Chapitre 1, "The frames of competition: Balzac, Grandville, and the caricature » (pp. 23-78 =55p.), Keri utilise les thèses de Bourdieu sur le champ culturel comme un champ de bataille sur lequel écrivains, éditeurs et critiques luttent pour conserver ou améliorer leur capital culturel et économique. Dans Les Français peints par eux-mêmes, Balzac et Grandville, semblent être à égalité mais en fait comme l'écrit Keri, « utilisée pour vendre des souscriptions et attirer les lecteurs comme voyeurs, l'image à laquelle toute autonomie artistique est refusée, devient en quelque sorte, la 'putain' de l'éditeur, sinon de l'écrivain.» (p.78, ma traduction $\left.{ }^{1}\right)$ C'est cette situation que 
Grandville n'acceptera pas comme le montre le chapitre suivant. En effet dans le Chapitre 2, "Caging the writer, Les Scènes de la vie privée et publique des animaux" (pp. 79-111 =31p.) l'auteur se concentre sur cette œuvre de 1840 citée dans le titre, et montre le mouvement de l'illustrateur vers l'autorat et la relégation de l'écrivain au rôle d'« illustrateur verbal » (p.88). Le Chapitre 3, «Penning the illustrator : caricature and illustration in La Comédie humaine " (pp. 113-31=18p) se penche sur la première édition de La Comédie humaine, où le personnage de Bixiou est utilisé pour contenir l'illustrateur et l'illustration à l'intérieur du cadre contrôlé par l'écrivain. Enfin dans le dernier chapitre, "The Illustrator unbound: Grandville's Un autre monde" (pp. 139-168 =29p), Keri montre comment l'artiste crée un langage hybride visuelverbal qui défie les frontières esthétiques de l'époque.

Dans sa courte mais excellente conclusion (pp.169-79, soit dix pages en tout), Keri prolonge sa démonstration critique sur la compétition entre image et texte à l'époque du Second Empire. Après le bref répit de 1848, la censure revient en force et l'illustration se scinde alors en deux domaines : l'illustration populaire et l'illustration de luxe. La première se fait de plus en plus légère, s'occupant principalement de mode et d'érotisme. Le personnage d'Anatole des frères Goncourt remplace le Bixiou de Balzac pour le pire : piètre artiste, peintre manqué, copieur, singe $(\mathrm{du})$ grotesque ; à cette première tendance s'ajoutent les manuels scolaires largement illustrés d'Hachette et de Larousse. L'illustration de luxe valorise les grands chefs d'œuvres, classiques surtout, source d'un investissement sûr, et parfois les bestsellers de l'époque. 1863 marque un nouveau tournant puisque c'est la date du Déjeuner sur l'herbe et d'Olympia, indiquant la prise de pouvoir des peintres sur la modernité : Paris, ses habitants, ses paysages et sa critique. Keri conclut avec Bourdieu que «ceci est un processus dynamique, une lutte entre des forces commerciales, esthétiques et techniques en compétition ». Il continue en écrivant que la collaboration-compétition entre Balzac et Grandville «documente un moment clé dans les arts et l'histoire du livre quand l'illustration modifia au moins temporairement les relations de pouvoir entre texte et illustration, écrivain et artiste, ouvrant et en fin de compte exposant les mécanismes du champ de production culturelle» (p.179; ma traduction $\left.{ }^{2}\right)$. Le texte est suivi d'un index complet et précis et d'une bibliographie qui reprend les nombreux textes cités en bas de page.

Cette étude est à lire en même temps que deux autres livres sur la caricature qui sont sortis récemment. L'un est publié en 2010, sous la direction de Dominique Avon (professeur d'histoire contemporaine, spécialiste des religions), intitulé La Caricature au risque des autorités politiques et religieuses (PUR), et qui traite en partie des " premières " caricatures de Mahomet (de 2006). L'autre date de février 2015, juste après et en quelque sorte en l'honneur des victimes du terrorisme islamiste de Charlie Hebdo, et s'intitule La Caricature... Et si c'était sérieux? Décryptage de la violence satirique (Nouveau Monde éditions) avec des textes brefs et brillants de huit spécialistes (Ory, Delporte, Matard-Bonucci, ...) et des reproductions en couleur de nombreuses caricatures dont certaines sexistes et racistes. Avec ces trois livres on peut se faire une bonne idée du rôle de la caricature et de l'image en France dans les deux derniers siècles. 


\section{NOTES}

1. "Used to sell subscriptions and entice readers voyeuristically, but denied artistic autonomy, the image becomes, in some sense, the publisher's, if not the writer's, whore" (p.78)

2. "As Pierre Bourdieu has argued, this is a dynamic process, art a struggle between competing aesthetic, technical, and commercial forces. [...] From challenge to codification, Grandville and Balzac - their collaboration, competition, and artistic trajectories - document a key moment in the arts and the history of the book when illustration temporarily altered power relations between text and illustration, writer and artist, pushing open and ultimately exposing the mechanisms of the field of cultural production." (p.179) 